\title{
Fibrotic lung toxicity induced by cytotoxic drugs, radiation and immunotherapy in patients treated for lung cancer
}

\author{
Elena Bargagli1, Viola Bonti', Alessandra Bindi2, Vieri Scotti³, Massimo Pistolesi', \\ Luca Voltolini4, Katia Ferrari1 \\ 1 Section of Respiratory Medicine, Department of Clinical and Experimental Biomedical Sciences, \\ University Hospital Careggi, Florence \\ 2 Radiology Unit, University Hospital Careggi, Florence \\ 3 Radiotherapy Unit, University Hospital Careggi, Florence \\ 4 Thoracic Surgery Unit, University Hospital Careggi, Florence, Italy
}

\begin{abstract}
Patients treated for lung cancer may develop lung toxicity induced by chemotherapy (DILD), radiation or combined radiation recall pneumonitis. In the literature, some cases of immune-mediated pneumonitis have been reported associated with immunotherapy. The clinical and radiologic features of interstitial lung toxicity are unspecific, dyspnea and dry cough are the most common symptoms while the most frequent radiological pattern is the cryptogenic organizing pneumonia (COP). Why only some individuals treated with these drugs develop interstitial lung toxicity is unclear. Old age, ethnicity, doses, drug interactions, oxygen damage and radiation therapy are known risk factors, as well as pre-existing lung disease. There are no clear indicators of the risk of developing lung toxicity secondary to drugs or radiation for individual patients. In the last few years some studies have reported the utility of KL 6 for the evaluation of DILD. The treatment is based on high doses of systemic steroids or immune suppressor. In this study we report severe interstitial lung damage in patients treated with different anti-blastic, immune and radiation therapies. Treated with surgery, chemotherapy, immuno- and radiotherapy for lung cancer, they unfortunately died of severe DILD.
\end{abstract}

Correspondence: Elena Bargagli, Department of Clinical and Experimental Biomedical Sciences, University Hospital Careggi, Largo Brambilla 1, 50134 Firenze, Italy. Tel. +39.339.1590310. E-mail bargagli2@gmail.com

Key words: Drugs; interstitial lung toxicity; interstitial pneumonitis; chemotherapy; oncological immunotherapy.

Received for publication: 22 February 2018

Accepted for publication: 22 May 2018

CC Copyright E. Bargagli et al., 2018

Tipografia PI-ME Editrice, Italy

Monaldi Archives for Chest Disease 2018; 88:917

doi: 10.4081/monaldi.2018.917

This article is distributed under the terms of the Creative Commons Attribution Noncommercial License (by-nc 4.0) which permits any noncommercial use, distribution, and reproduction in any medium, provided the original author(s) and source are credited.

\section{Introduction}

More than 350 different pharmacological molecules can provoke drug-induced respiratory disorders: lung toxicity may involve the airways, lung parenchyma, mediastinum, pleura, pulmonary vasculature and/or neuromuscular system. The most common manifestation of drug-induced lung toxicity is drug-induced interstitial lung diseases (DILD) [1-3]. Drugs frequently associated with the development of diffuse fibrotic diseases (more than $10 \%$ of patients exposed develop DILD) include cytotoxic drugs such as bleomycin, cyclophophamide, methotrexate and hydroxyurea. Many other drugs can adversely affect the lung, inducing different types of fibrotic alterations [1]. The principal risk factors for the development of DILD include age, sex, genetic factors, oxygen administration, drug interactions and dosages, radiation and pre-existing lung disorders [3]. In particular, patients treated for lung cancer may develop lung toxicity as a result of chemotherapy, radiation or combined therapies (radiation recall pneumonitis). The panorama of treatment options includes new immunotherapeutic drugs, approved for lung cancer therapy. These molecules enhance anti-tumor immunity by inhibiting negative $\mathrm{T}$ cell regulators and programmed cell-death-1 [4-10]. Immune checkpoint inhibitors currently approved for treatment of advanced NSCLC include nivolumab (anti CTLA-4 and anti PD1), pembrolizumab (anti PD1) and atezolizumab (anti PDL-1) [7,8]. Despite encouraging results, these drugs seem associated with autoimmune-like toxicities, such as pneumonitis, in $3-10 \%$ of cases $[9,11,12]$.

In this study we report three cases of severe interstitial lung damage in patients treated with different anti-blastic, immune and radiation therapies. Treated with surgery, chemotherapy, immuno- and radiotherapy for lung cancer, they unfortunately died of severe DILD.

\section{Case 1}

The first patient was a 72-year-old male, ex-smoker, with professional exposure to asbestos as a hairdresser (hairdryers once contained asbestos). In 2014, the patient manifested recurrent pleural effusion in the left lung, with negative histocytological assay. Two years later (May 2016) the patient was diagnosed with epitheliomorphic mesothelioma and underwent atypical resection of the upper and lower left lobes, pleural, pericardial, thymic and mediastinal lymph nodes. The patient underwent four cycles of chemotherapy with carboplatinum-pemetrexed until November 2016 and radiotherapy at $50 \mathrm{~Gy}$ in 25 fractions until February 2017. The patient was periodically assessed 
by oncologists and radiotherapists. Neoplastic disease was stable. However, in April 2017 the patient developed dry cough, dyspnea and bilateral edema of the legs, followed by respiratory failure and atrial fibrillation with concomitant heart failure. Fever was absent and infections were excluded by microbiological examination. HRCT of the chest revealed diffuse bilateral ground glass opacities, reticular fibrotic involvement and large areas of parenchymal consolidation (Figure 1). In the intensive care unit, the patient was treated with NIV/CPAP, optiflow, antibiotics (cotrimoxazole, piperacillin/tazobactam) and antimycotics (caspofungin), high dose iv steroids (methylprednisolone $250 \mathrm{mg}$ x 2, then $125 \mathrm{mg} / \mathrm{die}$ ) and oral azathioprine (50 $\mathrm{mg}$ every $12 \mathrm{~h}$ ). HRCT of the chest improved after one week of treatment with a significant reduction of ground glass opacities and interstitial involvement. The patient was transferred to our Pneumology Unit under oxygen therapy (ventimask Fi02 50-60\%), antibiotics and steroids. Azathioprine was stopped due to a sharp increase in creatinine levels. A few days later, the patient's condition deteriorated and HRCT revealed new bilateral ground glass opacities and areas of consolidation. He died of radiation recall pneumonitis two weeks later.

\section{Case 2}

The second patient was a 63-year-old male, ex-smoker, without professional exposure, with severe emphysema, COPD, GER and monoclonal gammopathy. He reported a recent increase in dry cough and dyspnea. Chest HRCT showed combined emphysema-fibrosis syndrome. A nodule was also detected in the right upper lobe associated with mediastinal lymph node involvement. PET was positive in these pathological areas and endobronchial ultrasound bronchoscopic biopsy revealed a lung adenocarcinoma TTF1+, EGFR and ALK WT, stage IIIB (cT2N3M0). After assessment by the multidisciplinary oncology team, cisplatinum plus gemcitabin therapy was administered from March to July 2016. In August 2016, PET showed improvement with weak uptake in the pulmonary and mediastinal areas. Maintenance therapy with gemcitabin (1 dose every 21 days) was introduced instead of radiotherapy, but in September 2016 the patient was hospitalized in the Emergency Unit with fever and severe respiratory failure. HRCT showed bilateral ground glass opacities and severe progression of the pre-existing fibrosis-emphysema pattern. Pulmonary embolisms and respira-

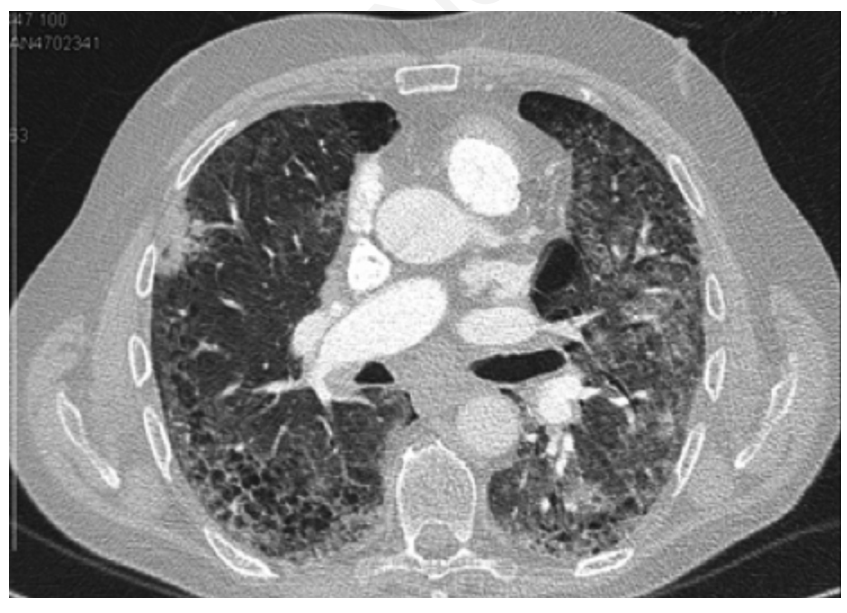

Figure. 1 High resolution CT of the chest revealed diffuse bilateral ground glass opacities, reticular fibrotic involvement and large areas of parenchymal consolidations. tory infections were excluded and the patient was treated with cPAP, high-dose systemic steroids and antibiotics. He died of severe rapid pulmonary fibrotic involvement ten days later.

\section{Case 3}

The third patient was a 72-year-old male, ex-smoker, ex electrician, with COPD, ischemic cardiopathy (treated with stenting in 2009) and squamous cell laryngeal carcinoma pT1N0 diagnosed in 2011. In January 2014 he underwent a follow up CT scan of the chest that detected a nodule in the lingular bronchus with stenosis and atelectasia. A bronchoscopy with TBNA was performed and squamous cell carcinoma was identified (lymph node $10 \mathrm{~L}$ positive). The total body CT scan was negative for metastases. PET evaluation revealed increased uptake in the left para-hilar lung. The patient was in stage IIb (cT2aN1M0) but it was decided to avoid surgery because of comorbidities; in June 2014 the patient started chemotherapy (carboplatinum + vinorelbine, four cycles) and concomitant radiotherapy in the mediastinal area (DTF 66 Gy). Tolerance was good and a reduction in bronchial stenosis was obtained. Unfortunately, six months later PET and CT scan showed recurrence of neoplastic disease in the left hilar area. In June 2015, the patient was treated with cyberknife radiotherapy but the response was unsatisfactory and compassionate use of Nivolumab was made to improve control of the disease. The patient started Nivolumab therapy and remained stable until March 2016, when after the eleventh cycle, CT scan revealed an increase in the left hilar lesion with pleural effusion. In May he was therefore treated with third line chemotherapy (carboplatinumtaxotere). After the first cycle, he complained of severe dyspnea and asthenia; blood gas analysis indicated hypoxemia. CT of the chest documented bilateral ground glass opacities and interstitial diffuse lung involvement with NSIP pattern. Infections were excluded by microbiological examination. Based on the radiological features and clinical symptoms of DILD, the patient was treated with high doses of systemic steroids. Unfortunately, interstitial involvement was completely refractory to the treatment (with radiological evidence of progressive development of what seemed to be UIP pattern). Respiratory exchange deteriorated sharply and the patient died in February 2017.

\section{Discussion}

Drug-induced interstitial lung disease is often implicated in lung toxicity and is a very heterogeneous group of disorders. The number of cytotoxic treatments and biological therapies that can cause fibrotic lung toxicity is increasing and 3\% of all cases of interstitial pneumonitis are secondary to exposure to drugs $[1,13]$. DILD can arise as a direct cytotoxic effect of a specific pharmacological compound or as a consequence of indirect release of various mediators of tissue damage (cytokines, chemokines, reactive oxygen species, etc.). Induction of alveolitis, pulmonary edema and aberrant fibrotic repair mechanisms lead to DILD [2]. Another pathogenetic mechanism of DILD may be oxidative burst and oxidant/antioxidant imbalance [3]. Generally, the histological and radiological patterns of DILD are unspecific and may include hypersensitivity pneumonitis, organizing pneumonia, usual interstitial pneumonia, diffuse alveolar damage, non-specific interstitial pneumonia, granulomatous pneumonitis, bronchiolitis obliterans organizing pneumonia [1-3].

In patients treated for lung cancer, radiation therapy may also induce fibrotic pulmonary damage, according to the site and extent of irradiation. It is estimated to occur in $5-10 \%$ of patients treated at mediastinal level and 5-50\% of those undergoing lung irradiation [14]. Associative 
treatment (radiation therapy combined with chemotherapy) may be synergistic in determining lung toxicity $[14,15]$. Radiation recall reaction is an inflammatory reaction arising in a previously irradiated area after application of precipitating agents, usually chemotherapeutic drugs. If the lungs are involved, radiation recall pneumonitis (RRP) may occur: radiotherapy presumably causes a permanent change in vascular permeability and in stem cells, leading to expression of cytokines that upregulate when a precipitating agent is introduced. Typical radiological patterns include ground glass opacities, diffuse haziness, infiltrations and consolidations [1-3,8]. Cases of pneumonitis in patients treated with immunotherapy after radiotherapy were recently reported by Shibaki et al.: two patients treated with radiotherapy 2 years earlier, developed pneumonia after four and six months of Nivolumab treatment [8]. In these cases, RRP exactly mapped the irradiated field and the interval between the end of radiotherapy and RRP was two years. In the literature, the time interval ranges from a few days to 9 months [8,13]; Ding et al. found a median time interval of 95 days [13]. Our third case developed RRP after chemotherapy and nivolumab, but lung involvement was bilateral, beyond the limits of the field irradiated.

The new immunotherapy molecules interfere with immunological tolerance mechanisms, inducing a response to lung cancer cells. They can have extraordinary clinical efficacy, unfortunately not without immune-related toxic effects [10-12]. Most data on the immune-related adverse effects of these drugs to the lungs comes from clinical trials conducted in the context of melanoma, NSCLC and renal cell carcinoma, while data and real-life results from clinical experience are extremely limited, despite ongoing clinical studies [8-11]. Although initial safety data has been published in the literature, an international multicenter clinical trial on 915 patients reported that pneumonitis developed in $5 \%$ of patients, with increased risk of lung toxicity in cases of combination therapy with anti CTLA-4 and anti PD-1 [8]. The safety data from this study offered interesting insights into the latency of onset: respiratory symptoms may occur in the first three months of therapy or up to 10 months after exposure. It is also suggested that rapid onset of DILD may carry a negative prognosis. The ILD associated with anti-programmed cell death 1 antibodies were reported by Naidoo et al. describing in particular the histories of three patients affected by melanoma and treated with nivolumab. A 70-year-old man who was treated in a trial of nivolumab given sequentially with ipilimumab. He developed bibasal consolidative areas with reticular and ground-glass opacities. He rapidly deteriorated with a progressive involvement of all the lungs. A 38-year-old woman treated with nivolumab for 4 months and developing wide bilateral diffuse ground-glass opacities, consolidations, nodules and traction bronchiectasis [8]. A 58-year-old man developing NSIP radiological pattern after only 7 weeks of therapy. Other data is provided by Nishino et al. who found that in a population of 170 patients treated with nivolumab, alone or in combination with other immune checkpoint inhibitors, 20 patients (11\%) developed pneumonitis [7]. The international clinical trials concluded low risk of DILD after treatment with nivolumab, pembrolizumab and docetaxel [8-12], but these results will presumably prove to be underestimated after 2 years follow-up.

Why only some individuals treated with these drugs develop interstitial lung toxicity is unclear. Old age, ethnicity, doses, drug interactions, oxygen damage and radiation therapy are known risk factors, as well as pre-existing lung diseases [1,3]. There are no clear indicators of the risk of developing lung toxicity secondary to drugs or radiation for individual patients. In the last few years some studies have reported the utility of KL 6 for the evaluation of DILD. KL 6 is typically elevated in patients with idiopathic interstitial pneumonia and in certain cases of cancer, and may therefore be useful for evaluating disease activity and clinical outcome in patients with ILD-IPF: it could predict the likelihood of radiation pneumonitis or lung toxicity induced by chemotherapy [15-20]. Recently, a similar predictive role of Surfactant Protein D (SPD) for onset of ILD induced by anticancer agents was compared with KL 6 in an interesting case control study [18]. Nakamura et al. also reported that SP-D was associated with worse prognosis and development of diffuse alveolar damage [18].

Other interesting studies describe the histotypes and the morphological and molecular features of lung cancer in patients with pulmonary fibrosis: in contrast with previous studies, they report a higher percentage of adenocarcinoma in cases with fibrosis having certain molecular features [5]. Another article described the frequency of EGFR mutation, ALK and ROS1 rearrangement and PDL 1 expression in patients with fibrosis and lung cancer [19-21]. Although conducted on limited cohorts, these studies suggest the need for more detailed molecular characterization of every type of lung fibrosis associated with cancer in order to define potential therapeutic targets. These findings imply that similar molecular-immunochemistry studies and biomarkers may be useful to predict the risk of developing ILD for patients treated for lung cancer. In our opinion, patients should be monitored closely for toxicity, which may arise at any stage during treatment.

\section{References}

1. Schwaiblmair M, Behr W, Haeckel T, et al. Drug induced interstitial lung disease. Open Resp Med J2012;6:63-74.

2. Camus P, Foucher P, Bonniaud P, Ask K. Drug-induced infiltrative lung disease. Eur Respir J 2001;18:93-100s.

3. Camus P, Bonniaud P, Fanton A, et al. Drug- induced and iatrogenic infiltrative lung diseases. Clin Chest Med 2004;5:479-519.

4. Guyard A, Danel C, Théou-Anton N, et al. Morphologic and molecular study of lung cancers associated with idiopathic pulmonary fibrosis and other pulmonary fibrosis. Respir Res 2017;18:120-6.

5. Caliò A, Lever V, Rossi A, et al. Increased frequency of bronchiolar histotypes in lung carcinomas associated with idiopathic pulmonary fibrosis. Histopathology 2017;7:725-35.

6. Nishino M, Ramaiya N, Awad M, et al. PD-1 inhibitor-related pneumonitis in advanced cancer patients: Radiographic patterns and clinical course. Clin Cancer Res 2016;22:6051-60.

7. Nishino M, Chambers ES, Chong CR, et al. Anti-PD-1 inhibitorrelated pneumonitis in non-small cell lung cancer. Cancer Immunol Res 2016;4:289-93.

8. Naidoo J, Wang X, Woo KM, et al. Pneumonitis in patients treated with anti-programmed death-1/programmed death ligand 1 therapy. J Clin Oncol 2017;35:709-17.

9. Reck M, Abreu M, Robinson A, et al. Pembrolizumab versus chemotherapy for PD-L1-positive non-small-cell lung cancer. N Engl J Med 2016;375:1823-33.

10. Brahmer J, Reckamp KL, Baas P: Nivolumab versus docetaxel in advanced squamous-cell non-small-cell lung cancer. N Engl J Med 2015;373:123-35.

11. Feld E, Horn L. Emerging role of nivolumab in the management of patients with non-small cell lung cancer: current data and future perspectives. Onco Targets Ther 2017;10:3697-708.

12. Shibaki r, Akamatsu H, Fujimoto M, et al. Nivolumab induced radiation recall pneumonitis after two years of radiotherapy. Ann Oncol 2017;28:1404-5.

13. Ding X, Ji W, Li J, et al. Radiation recall pneumonitis induced by chemotherapy after thoracic radiotherapy for lung cancer. Radiat Oncol 2011;6:24. 
14. Azria D, Magné N, Zouhair A, et al. Radiation recall: a well recognized but neglected phenomenon. Cancer Treat Rev 2005;31:555-70.

15. Friedman C, Singh T, Postow M. Treatment of the immune-related adverse effects of immune check point inhibitors. A review. JAMA 2016;2:1346-53.

16. Sweis RF, Luke JJ. Mechanistic and pharmacologic insights on immune checkpoint. Pharmacol Res 2017;120:1-9.

17. Saynack M, Higginson D, Morris DE, Marks LB. Current status of postoperative radiation for non small cell lung cancer. Semin Radiat Oncol 2010;20:192-200.

18. Nakamura K, Kato M, Shukuya T, et al. Surfactant protein-D predicts prognosis of interstitial lung disease induced by anticancer agents in advanced lung cancer: a case control study. BMC Cancer 2017;17:302-11.

19. Wakamatsu K, Nagata N, Kumazoe H, et al. Prognostic value of serial serum KL6 measurements in patients with idiopathic pulmonary fibrosis. Respir Investig 2017;55:16-23.

20. Hara R, Itami J, Komiyama T, et al. Serum levels of KL 6 predicting the occurrence of radiation pneumonitis after stereotactic radiotherapyfor lung tumors. Chest 2004;125:340-4.

21. Kashiwabara K, Semba H, Fujii S, et al. The ratio KL6 to SLX in serum for prediction of the occurrence of drug induced interstitial lung disease in lung cancer patients with idiopathic interstitial pneumonias receiving chemotherapy. Cancer Investig 2015;26:516-21. 